\title{
Increased-Resolution OCT Thickness Mapping of the Human Macula: A Statistically Based Registration
}

\author{
Rui Bernardes, ${ }^{1,2}$ Torcato Santos, ${ }^{1}$ and José Cunba-Vaz ${ }^{1,3}$
}

Purpose. To describe the development of a technique that enhances spatial resolution of retinal thickness maps of the Stratus OCT (Carl Zeiss Meditec, Inc., Dublin, CA). A retinal thickness atlas (RT-atlas) template was calculated, and a macular coordinate system was established, to pursue this objective.

Methods. The RT-atlas was developed from principal component analysis of retinal thickness analyzer (RTA) maps acquired from healthy volunteers. The Stratus OCT radial thickness measurements were registered on the RT-atlas, from which an improved macular thickness map was calculated. Thereafter, Stratus OCT circular scans were registered on the previously calculated map to enhance spatial resolution.

Results. The developed technique was applied to Stratus OCT thickness data from healthy volunteers and from patients with diabetic retinopathy (DR) or age-related macular degeneration (AMD). Results showed that for normal, or close to normal, macular thickness maps from healthy volunteers and patients with DR, this technique can be an important aid in determining retinal thickness. Efforts are under way to improve the registration of retinal thickness data in patients with AMD.

Conclusions. The developed technique enhances the evaluation of data acquired by the Stratus OCT, helping the detection of early retinal thickness abnormalities. Moreover, a normative database of retinal thickness measurements gained from this technique, as referenced to the macular coordinate system, can be created without errors induced by missed fixation and eye tilt. (Invest Ophthalmol Vis Sci. 2008;49:2046-2052) DOI: 10.1167/iovs.07-0467

$\mathrm{T}$ he purpose of this report is to describe the benefits of optical coherence tomography (OCT) macular mapping of the human retina.

Macular edema, which is characterized by an increase in retinal thickness involving the macula, ${ }^{1}$ is a frequent manifestation of diabetic retinopathy (DR) ${ }^{2}$ and age-related macular degeneration (AMD) and is a leading cause of legal blindness in patients with type 2 diabetes. $^{3}$ Monitoring and mapping ${ }^{1}$ changes in macular edema over time have provided valuable

From the ${ }^{1}$ Centro de Novas Tecnologias para a Medicina da Associação para a Investigação Biomédica e Inovação em Luz e Imagem (AIBILI/CNTM), Coimbra, Portugal; and the ${ }^{2}$ Instituto de Biofísica e Biomatemática, and the ${ }^{3}$ Centro de Oftalmologia, IBILI, Faculdade de Medicina, Universidade de Coimbra, Coimbra, Portugal.

Supported by EVIGeM project (European Virtual Institute for Geometry Measurement) Contract G7RT-CT-2002-05086 and EVI-GENORET (European Vision Institute-Functional Genomics of the Retina in Health and Disease) Contract LSHG-CT-2005-512036).

Submitted for publication April 19, 2007; revised July 16, September 3, October 19, and November 28, 2007; accepted March 5, 2008 Disclosure: R. Bernardes, None; T. Santos, None; J. Cunha-Vaz, None

The publication costs of this article were defrayed in part by page charge payment. This article must therefore be marked "advertisement" in accordance with 18 U.S.C. $\$ 1734$ solely to indicate this fact.

Corresponding author: Rui Bernardes, AIBILI/IBILI, Azinhaga Sta Comba, Celas, 3000-548 Coimbra, Portugal; rcb@aibili.pt. information to assist in making clinical healthcare decisions. ${ }^{4-6}$ Moreover, monitoring macular edema has been shown to be a useful practice in other circumstances, such as after cataract surgery. ${ }^{7}$

The evaluation of retinal thickness has been performed traditionally by stereofundus photography (SFP) and, more recently, by OCT models 2 and Stratus (Carl Zeiss Meditec, Inc., Dublin, CA) and RTA I and II (Retinal Thickness Analyzer; Talia Technology, Lod, Israel).

Macular edema assessment by SFP consists of imaging the retina in stereo by taking photographs of the ocular fundus from two different viewpoints. Thereafter, an expert in analyzing the results looks at those images with the use of stereoscopic viewers to assess any increase in retinal thickness from the normal pattern. SFP therefore represents a qualitative assessment of retinal thickness, including the subjectivity of such an evaluation while establishing several grades of retinal thickness. In contrast, both OCT and RTA represent quantitative assessments of retinal thickness, thus making possible the objective measurement of retinal edema or the increase in retinal thickness from a healthy reference population, while removing the subjectivity inherent in the SFP technique.

Several reports in the literature have established comparisons between these qualitative and quantitative assessment techniques. Most have demonstrated the advantages of the quantitative over the qualitative approach. ${ }^{5,8-21}$

Although both OCT and RTA are quantitative techniques, the biggest disadvantage of OCT is the poor resolution of the retinal thickness maps that it produces. This weakness has not been addressed in any model from the classic to the Stratus OCT version and therefore remains to be resolved. Until now, OCT retinal thickness maps could not be built by accounting for their relative positions, and their location within the retinal area has not been factored into the evaluation.

In this report, we address these problems while describing a technique that increases the resolution of macular thickness maps. Increased resolution can be achieved by integrating different scan types-specifically, radial and circular scans. Simultaneously, the relative position between scans and the center of the fovea can be estimated. The entire process can be achieved by registering radial and circular scan sets, as well as their individual components, into an atlas template of human retinal thickness, which was established to conduct this work without the use of ocular fundus references. In this context, atlas refers to a template representing the average geometry of a sample of individuals, a common definition in the human brain mapping community. ${ }^{22}$

Prior work in this field was performed by our group in 2003 and 2004 (Bernardes et al. IOVS 2003;44:ARVO E-Abstract 4852; Bernardes et al. IOVS 2004; 45 :ARVO E-Abstract 2368). Our initial approach (2003) allowed the integration of any line scans in a user-assisted registration mode, making use of the fundus image provided by OCT. In 2004, a new procedure for the Stratus OCT allowed the integration of line and circle scans, using a data-driven registration by searching for the best match at scan intersections. Although both methods registered OCT scans among themselves, none took into account the location of the scans within the macular area. In 2005, Soer- 
ensen et al. (IOVS 2005; 46:ARVO E-Abstract 2574) followed a different approach by merging two sets of radial line scans, therefore decreasing the step angle from $30^{\circ}$ to $15^{\circ}$. To our knowledge, no attempt was made to correct for the location or relative location of the scans, which were thought to cross at the center of the fovea. Finally, in 2006, our group presented the final version of the method, which is described herein in detail (Baptista et al., IOVS 2006;47:ARVO E-Abstract 5728).

All steps presented throughout the report address the aforementioned problems. In the Methods section we detail the instrumentation used, the process of registering RTA maps among themselves, how to build the atlas template, how to register OCT scans on the atlas template, and how to achieve increased map resolution. The tests performed with the developed system by using data from healthy volunteers, patients with DR, and patients with AMD are specified. In the analysis section we also define the quantitative and qualitative grading system to be used.

\section{MeTHODS}

We used an atlas of the human retinal thickness to register OCT scans from two acquisition protocols.

The technique was tested in a group of healthy volunteers and in patients with diagnosed DR or AMD. Approval was obtained from the local institutional review board, and all subjects gave their informed consent before they were enrolled in the study, in compliance with the tenets of the Declaration of Helsinki.

\section{Instrumentation}

OCT macular thickness mapping is based on six radial-line scans (B-scans) taken in 1.92 seconds using the Fast Macular Protocol. The angle between consecutive scans is $30^{\circ}$; hence, a large interpolation is achieved when mapping retinal thickness, with the interpolation factor increasing with the distance to the center of the map. Moreover, all six radial-line scans are expected to cross at the center of the map and at the center of the fovea, but do not correct for eventual misfixations and/or difficulties in focusing the target area. ${ }^{14,15,17}$

In our study, two protocols were used to collect retinal thickness information with the Stratus OCT: the Fast Macular Protocol and the Fast RNFL Protocol. Whereas the former consists of six-radial line scans, $30^{\circ}$ apart and $6000 \mu \mathrm{m}$ in length (default), the latter consists of six-concentric circle scans having radii of 1440, 1690, 1900, 2250, 2730 , or $3400 \mu \mathrm{m}$. Each line component of the Fast Macular Protocol and each circle component of the Fast RNFL Protocol are composed of 128 retinal thickness measurements.

In contrast, the RTA (version II) is a slit lamp-based system that scans five areas of $3 \times 3 \mathrm{~mm}^{2}$ each, with four areas side-by-side and the fifth overlapping the others and centering on the fovea.

The RTA system uses a vertical narrow green $\mathrm{HeNe}(543 \mathrm{~nm})$ laser slit beam projected obliquely, and backscattered light is recorded by a charged-coupled device. Because of the oblique projection, backscattered light returns two peaks, one corresponding to the vitreoretinal interface and the other corresponding to the chorioretinal interface. The distance between these two peaks is the measured retinal thickness at that specific location in the eye.

For each of the five scanned areas, 16 optical cross-sections are analyzed to compute the thickness of the retina by measuring the distance between the two detected peaks. The thickness information is then assembled into a final retinal thickness map.

\section{Retinal Thickness Atlas}

To establish the retinal thickness atlas (RT-atlas), retinal thickness data are collected with the RTA II from 40 eyes of 20 healthy volunteers, aged from 40 to 64 years (mean \pm SD: $52.4 \pm 5.9$ years). Each RTA map was individually analyzed to check for alterations from normal appear- ance. Eight eyes were eliminated in this way, leaving 32 eyes to be considered.

Atlas construction for medical purposes has been a research field of its own. ${ }^{23-25}$ In this application, atlas construction followed a mixed model in which all individual RTA maps were initially registered into the atlas space. Added to this are 32 RTA maps that were individually registered thereafter to subpixel accuracy. From this second registration process, the final RT-atlas was established.

To build the RT-atlas, we initially brought each RTA map into alignment and then rotated it (based on the fundus image reference available on the RTA) so that the line connecting the center of the fovea to the center of the optic disc lay in the horizontal axis. The maps are brought into alignment by fitting each one with a paraboloid, as in equation 1.

$$
f(x, y)=a+b\left(\alpha\left(x-x_{0}\right)^{2}+\beta\left(y-y_{0}\right)^{2}\right)^{c} .
$$

From the fitted parameters $\left(a, b, c, \alpha, \beta, x_{0}, y_{0}\right)$, the center of the paraboloid allows the translation to be computed $\left(-x_{0},-y_{0}\right)$, which brings the foveal depression of the respective RTA map into the origin of the atlas coordinate system in the $x-y$ plane.

The rotation angle, on the other hand, is computed by manually pointing and clicking at the optic disc center, while the center of the fovea is detected, as mentioned.

That is, we consider a rigid transformation $T_{i}$ for each map such that

$$
\tilde{r}_{i}=f\left(T\left(r_{i}\right)\right)
$$

where $r_{i}$ is the retinal thickness map $i(i=1 \ldots K), T_{i}$ is the corresponding rigid transformation (as defined in equation 3 ), $f$ is a function specifying a bicubic interpolation spline to be sampled into a common grid spacing for the set of $\tilde{r}_{i}$ maps, and $\tilde{r}_{i}$ is the translated, rotated, and resampled retinal thickness map to be used to establish the RT-atlas.

$$
T_{i}=\left[\begin{array}{ccc}
\cos (\theta) & -\sin (\theta) & -x_{0} \\
\sin (\theta) & \cos (\theta) & -y_{0} \\
0 & 0 & 1
\end{array}\right]
$$

The asymmetry of the retinal nerve fiber layer (RNFL) distribution must be accounted for, to build an atlas based on both left and right eyes. Therefore, a right eye atlas is built by horizontally flipping left eye $\tilde{r}_{i}$ maps relative to the origin $(0,0)$. In this way, the built RT-atlas not only establishes a coordinate system, with the origin being the center of the fovea and the horizontal axis the line connecting both the center of the fovea and optic disc, but it also establishes that positive values in the $x$-axis (the horizontal axis, connecting the fovea to the optic disc) correspond to the nasal macula (fovea-wise) and negative values correspond to the temporal macula (fovea-wise). Similarly, the $y$-axis (the vertical axis) establishes (fovea-wise) negative and positive values as being in the superior and inferior macula, respectively.

Principal component analysis (PCA) is a useful statistical technique, with application in a large number of fields such as pattern recognition and image compression. It is commonly used for finding patterns in data of high dimension, and can be applied to the stack of our $\tilde{r}_{i}$ thickness maps, with each map considered to be an independent variable. Hence, for each coordinate in the space defined by transformation (equation 2), a set of thickness data exists.

PCA identifies a linear transformation of the coordinate system, such that the axes of the new coordinate system come into alignment with the directions of the $K$ largest spreads of the cluster, all being orthogonal among themselves and therefore being the data uncorrelated in this new set of axes. ${ }^{26}$

This transformation can be found by computing an $A$ matrix composed of the eigenvectors of the covariance matrix $(C)$ of the original data. This covariance matrix is built by considering each retinal thick- 
ness map as an independent variable with the dimension $K \times K$ and is computed as

$$
C(i, j) \equiv E\left\{\left(x_{i}-\bar{x}_{i}\right)\left(x_{j}-\bar{x}_{j}\right)\right\}
$$

where $x_{m}$ and $\bar{x}_{m}$ are, respectively, the retinal thickness and the average retinal thickness of map $m$, and $E\{\cdot\}$ is the expectation.

By computing vectors $\mathbf{v}$ and scalars $\lambda$, respectively, eigenvectors and eigenvalues, that is, the nontrivial solution of

$$
C \mathbf{v}=\lambda \mathbf{v}
$$

the transformation matrix $A$ is given by

$$
A=\left[\begin{array}{ccc}
\mathbf{v}_{11} & \ldots & \mathbf{v}_{1 K} \\
\vdots & \ddots & \vdots \\
\mathbf{v}_{K 1} & \ldots & \mathbf{v}_{K K}
\end{array}\right]
$$

where matrix $A$ line $i$ is the transpose of vector $\mathbf{v}_{i}$ (eigenvector $i$ ) to which corresponds the eigenvalue $\lambda_{i}$, being $\lambda_{1} \geq \lambda_{2} \geq \ldots \geq \lambda_{K} \geq 0$.

The eigenvectors of the covariance matrix $C$ form an optimal orthogonal basis for the entire set of retinal thickness maps. Because the set of retinal thickness maps (i.e., multiple views of retinas of healthy volunteers), is correlated, the vector associated with the larger eigenvalue represents the entire set of information with the least expansion error compared with other orthogonal expansions.

The initial RT-atlas can therefore be made equal to the first PCA,

$$
\mathrm{PCA}_{1}=\sum_{i=1}^{K} \mathbf{v}_{1 i} \tilde{r}_{i}
$$

This RT-atlas has a standard variation $(\sigma)$ of $\sqrt{\lambda_{1}}$, and thus the largest spread of data from the ensemble of PCAs $\left(\lambda_{1} \geq \lambda_{2} \geq \ldots \geq \lambda_{K} \geq 0\right)$.

To refine this model, we registered each of the $K$ original retinal thickness maps in the established $P C A_{1}$, resorting to an optimization process, and repeated the procedure, with the PCA now applied to compute a new set of eigenvectors and eigenvalues. The final RT-atlas (Fig. 1) was computed as before, with all maps registered to subpixel accuracy to the initial atlas $\left(P C A_{1}\right)$.

The difference between the initial $\left(P C A_{1}\right)$ and the final (RT-atlas) computed atlases, after their normalization to the range $(0,1)$, is $3.2 \%$. Five RTA maps were rejected due to the application of a validation criterion to the estimated parameters $(x, y$ for translation and $\theta$ for rotation); that is, a map was rejected if any one of these was not within 1.75 standard deviations from its mean, which accounts for more than $90 \%$ of the cases for a normal distribution.

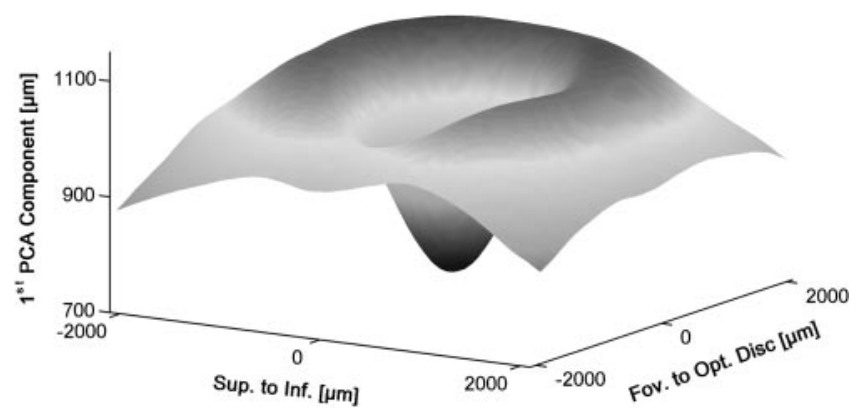

FigURE 1. 3D plot of the RT-atlas (a statistical shape model/template) in a macular coordinate system. This RT-atlas represents the average shape of a healthy retinal thickness but not the average thickness.

\section{OCT to RT-atlas Registration}

To find where in the retina a given OCT scan is performed, a match can be sought by comparing the OCT scan with the RT-atlas, centered at any position and having any direction (line scans). The best-fitting profile shape is, therefore, the preferred candidate for establishing the location and angle where the OCT scan was performed, in RT-atlas coordinates, thus establishing absolute coordinates in the RT-atlas space for each OCT scan.

In this study we restricted the search space by registering the Fast Macular Protocol into the RT-atlas, because individual scans belonging to this protocol share a common global location. Although different scans can be found centered at different locations, they should all be within a certain distance due to the acquisition speed. We made use of this additional information for the registration procedure, which was performed in several consecutive steps as the number of optimization parameters, and their interrelation added difficulty to their simultaneous estimation.

Even though saccades introduced some distortions in scan paths, this effect was not taken into account in the Fast Macular Protocol scans; the registration procedure looked for the best match within a line in the RT-atlas.

Before the optimization procedure, all OCT scans were filtered by a Gaussian low-pass filter, with normalized cutoff frequency of $0.132 \pi$ $\mathrm{rad} / \mathrm{sample}$, to prevent the existence of local minima. Nevertheless, for thickness mapping only measured thickness values were used.

Our next goal was to estimate $\Gamma$, which minimized the total registration error for the entire set of OCT line scans.

For the initial optimization step, all six radial line scans composing the Fast Macular Protocol were considered as a single entity and were allowed to move in the RT-atlas space to find the position that minimized their total error. Because we were interested in mapping macular edema, we started by considering that the scan set was centered at the fovea (i.e., at the origin of the RT-atlas space).

The initial optimization step consists of finding translation parameters in the RT-atlas space, in the $x$-and $y$-axes, that minimize the error (err) given by

$$
\operatorname{err}=\frac{1}{N} \sum_{i=1}^{N} E\left\{\Omega \cdot\left(O_{i}-\mathbf{R}\right)^{2}\right\}
$$

where $N=6$ is the number of radial scans, $O_{i}$ is the low-pass filtered thickness measurements of OCT scan $i, \mathbf{R}$ is a thickness vector computed from the RT-atlas, and $\mathrm{E}\{\cdot\}$ is the expectation of the dot product between $\Omega$ (a vector of weights) and the squared differences between $O_{i}$ and $\mathbf{R}$ on their overlapping areas.

We intended therefore to estimate $\Gamma_{1}=(x, y)$ such that

$$
\hat{\Gamma}_{1}=\arg \min _{\Gamma_{1}} \frac{1}{N} \sum_{i=1}^{N} E\left\{\Omega \cdot\left(O_{i}-\mathbf{R}\right)^{2}\right\}
$$

choosing as the starting point $(0,0)$ and subject to $|x| \leq \delta_{1}$ and $|y| \leq \delta_{1}$.

For the second step, we increased the dimension of $\Gamma_{1}$ by adding a new degree of freedom that allows the scan set to rotate as a whole, while simultaneously fine tuning its global position based on the previous optimization step. That is, we wanted to estimate $\Gamma_{2}=(x, y, \theta)$ with $\left(x_{0}, y_{0}\right)$, the starting point, made equal to $\hat{\Gamma}_{1}$ and $\theta_{0}=0$. Hence, in this second optimization step, both the global position and global rotation adjusted themselves. For this step, the constraint $|\theta| \leq \delta_{2}$ was added.

Having now found both the global position and orientation, $(\hat{x}, \hat{y})$, and $\hat{\theta}$, respectively, we performed additional steps to accommodate the differences in instrumentation used to establish the RT-atlas and to perform the scan: the RTA II and Stratus OCT. 
Three new parameters were considered: a $D C$ component $(d c)$ to accommodate an average difference between retinal thickness measurements, a gain factor $(g)$ to accommodate for different retinal thickness measurements, and a sampling rate (spacing) factor ( $s r$ ) to accommodate the difference in the sample spacing.

In this new optimization step, we estimated $\Gamma_{3},=(d c, g, s r)$ with $\left(d c_{0}, g_{0}, s r_{0}\right)=(0,1,1)$ and $\hat{\Gamma}_{2}$, the result of the previous optimization step. Constraints also applied, with $|d c| \leq \delta_{3}, \delta_{4} \leq g \leq \delta_{5}$, and $\delta_{6} \leq$ $s r \leq \delta_{7}$.

By following these three steps, we registered the Fast Macular Protocol scan (as a single entity) into the RT-atlas and estimated parameters to accommodate for differences in instrumentation.

New degrees of freedom are now considered to fit individually each of the scans composing the Fast Macular Protocol around the estimated parameters for the entire set. For each radial line scan, three degrees of freedom were considered (i.e., $\Gamma_{4}^{i}=\left(\Delta x_{i}, \Delta y_{i}, \Delta \theta_{i}\right)$ with $\left.i=1 \ldots N\right)$. Therefore, we had to perform $N$ individual optimizations; that is, for each one, we had to estimate $\Gamma_{4}^{i}$ subject to $\left|\Delta x_{i}\right| \leq \delta_{8},\left|\Delta y_{i}\right| \leq \delta_{8}$, and $\left|\Delta \theta_{i}\right| \leq \delta_{9}$.

After this sequence of steps, we solved the global optimization parameter $\Gamma$ of the type

$$
\Gamma=\left(x, y, \theta, d c, g, s r, \Delta x_{1}, \Delta y_{1}, \Delta \theta_{1}, \ldots, \Delta x_{N}, \Delta y_{N}, \Delta \theta_{N}\right) .
$$

Having estimated $\Gamma$, it was possible to build a retinal thickness map based on the Fast Macular Protocol data. Relative to the original map built by the Stratus OCT, this new map presents three major advantages. First, this mapping system does not assume that all radial line scans intersect at the same point. Second, because OCT scans are brought into registration to the RT-atlas space, they possess absolute RT-atlas coordinates. Therefore, independent of misfixations, the fovea will be centered at the origin.

A third advantage is in having the line connecting the center of the fovea to the center of the optic disc lying in the horizontal axis. This feature improves the establishment of OCT normative data by correcting for eye tilts.

To build the retinal thickness map based on the established registration of OCT scanned data, we can use a thin-plate spline (TPS) ${ }^{27}$ with the OCT-registered data points as control points. This approach allows defining a surface passing through every control point while presenting the least bent surface for the entire space (minimum bending energy surface)

\section{Increasing Mapping Resolution}

The previous section describes the registering of OCT scans performed by the Fast Macular Protocol in the RT-atlas space. In this section, we indicate how to increase the map's resolution (i.e., build a retinal thickness map with increased density of Stratus OCT thickness measurements).

Similar to the Fast Macular Protocol, we used data from the Fast RNFL Protocol. Several steps considering both global parameters were given for the set of scans performed by this protocol, and individual parameters were given for each circular scan.

The choice for this type of OCT scan is the same that led us to choose the Fast Macular Protocol in the previous section (i.e., all scans from the Fast RNFL Protocol share a common global location). On the other hand, although previously we did not account for distortions in scan paths due to saccades, these effects were considered here, because we were registering retinal scans on a retinal thickness map of the same eye.

Similar to the procedure followed for Fast Macular Protocol scans, Fast RNFL Protocol scans were also low-pass filtered to avoid local minima.

Although Fast Macular Protocol scans were registered on the RTatlas, Fast RNFL Protocol scans were registered on the built retinal thickness map but not on the RT-atlas, which is the major difference between procedures. Our goal was to estimate $\Psi$, which minimizes the total registration error for the entire set of OCT scans performed by Fast RNFL Protocol. For the same reasons as before, we start by considering that the Fast RNFL Protocol was performed centered in the fovea.

We intended, therefore, to estimate $\Psi_{1}=\left(x_{\mathrm{c}}, y_{\mathrm{c}}\right)$ to minimize the error given by

$$
\mathrm{err}=\frac{1}{M} \sum_{i=1}^{M} E\left\{\left(O_{i}-\mathbf{R}^{\prime}\right)^{2}\right\}
$$

where $M$ is the number of circular scans, $O_{i}$ is the low-pass filtered thickness measurements of OCT scan $i, \mathbf{R}^{\prime}$ is a thickness vector computed from the OCT map built based on the Fast Macular Protocol scans (the TPS surface of the previous section), and $E\{\cdot\}$ is the expectation of the squared differences between $O_{i}$ and $\mathbf{R}^{\prime}$ on their overlapping areas.

It should be noted that the circular scan with a $3400-\mu$ m radius was not considered as it corresponded to retinal thickness measurements beyond the limits of the previous retinal thickness map, as Fast Macular Protocol scans are $6000 \mu \mathrm{m}$ in length.

Hence, we estimated $\Psi_{1}=\left(x_{\mathrm{c}}, y_{\mathrm{c}}\right)$ as

$$
\hat{\Psi}_{1}=\arg \min _{\Psi_{1}} \frac{1}{M} \sum_{i=1}^{M} E\left\{\left(O_{i}-\mathbf{R}^{\prime}\right)^{2}\right\}
$$

choosing $(0,0)$ as a starting point, subject to $\left|x_{c}\right| \leq \gamma_{1},\left|y_{c}\right| \leq \gamma_{1}$, and $M=5$.

The need to estimate $\Psi_{1}$ instead of using $\hat{\Gamma}_{1}$, comes from the fact that the Fast Macular and Fast RNFL Protocols are performed independently, which means two acquisitions at two instances in time, therefore eliminating values computed for the Fast Macular Protocol.

The second step for the Fast RNFL Protocol is the same as the one performed for the Fast Macular Protocol (i.e., adding a rotation parameter to $\Psi_{1}$ to become $\Psi_{2}=\left(x_{\mathrm{c}}, y_{\mathrm{c}}, \theta_{\mathrm{c}}\right)$. Similarly, both the global position and global rotation are left to adjust themselves. For this step the constraint $\left|\theta_{c}\right| \leq \gamma_{2}$ is added.

Since we were registering OCT measurements on a map built on OCT measurements (i.e., similar data from the same instrumentation), there was no need to compute scaling parameters as before.

We let each circular scan fit itself around the established parameters for the entire set, in a manner similar to that for the Fast Macular Protocol.

Therefore we performed $M$ individual optimizations, whereby we estimated $\Xi_{i}^{1}=\left(\Delta x_{c i}, \Delta y_{c i}, \Delta \theta_{c i}\right)$ with $i=1 \ldots M$, subject to $\left|\Delta x_{c i}\right|$ $\leq \gamma_{3},\left|\Delta y_{c i}\right| \leq \gamma_{3}$, and $\left|\Delta \theta_{c i}\right| \leq \gamma_{4}$ for each scan.

Steps given so far correspond to those given for the Fast Macular Protocol. Because we were considering path deformations due to saccades, additional degrees of freedom were introduced.

Two new registration steps were performed. The first considered transforming circular scans into ellipses. This optimization step consisted of estimating $\Xi_{i}^{2}=\left(r_{x i}, r_{y i}, \varepsilon_{i}\right)$ subject to $\gamma_{5} \leq r_{x i} \leq \gamma_{6}, \gamma_{7} \leq r_{y i}$ $\leq \gamma_{8}$, and $\gamma_{9} \leq \varepsilon_{i} \leq \gamma_{10}$.

The second registration step allows ellipses to undergo shear and scale transformations with independent scales on the $x$ - and $y$-axes, consisting of estimating $\Xi_{i}^{3}=\left(\boldsymbol{s}_{x i}, \boldsymbol{s}_{y i}, \boldsymbol{s}_{b x i}, \boldsymbol{s}_{b y i}\right)$ subject to $\gamma_{11} \leq \boldsymbol{s}_{x i} \leq \gamma_{12}$, $\gamma_{13} \leq s_{y i} \leq \gamma_{14}, \gamma_{15} \leq s_{b x i}, \leq \gamma_{16}$, and $\gamma_{17} \leq s_{b y i}, \leq \gamma_{18}$.

These steps solve the global optimization parameter $\Pi$ of the type

$$
\begin{gathered}
\prod=\left(x, y, \theta, \Delta x_{1}, \Delta y_{1}, \Delta \theta_{1}, \ldots, \Delta x_{N}, \Delta y_{N}, \Delta \theta_{N}, \ldots\right. \\
\ldots, x_{c}, y_{c}, \theta_{c}, \Delta x_{c 1}, \Delta y_{c 1}, \Delta \theta_{c 1}, \ldots, \Delta x_{c M}, \Delta y_{c M}, \Delta \theta_{c M}, \ldots . \\
\left.r_{x 1}, r_{y 1}, \varepsilon_{1}, \ldots, r_{x M}, r_{y M}, \varepsilon_{M}, s_{x 1}, s_{y 1}, s_{b x 1}, s_{b y 1}, \ldots, s_{x M}, s_{y M}, s_{b x M}, s_{b y M}\right) .
\end{gathered}
$$

In this way, the sampling increases from a total of $768(6 \times 128)$ to $1408(11 \times 128)$ data points (i.e., $>1.8$ times the original sampling). 
Still, more important than the number of data points is the data distribution over the macular area, which is achieved by mixing data from the Fast Macular Protocol and Fast RNFL Protocol.

The final OCT retinal thickness map is therefore computed through the use of a TPS, according to the same approach as in the previous section.

\section{Tests and Grading}

The system was tested in three different groups of eyes. Group 1 is composed of 12 eyes of six healthy volunteers, aged 21 to 40 years (mean \pm SD: $28.0 \pm 6.69$ years), with normal retinas. Group 2 is composed of 18 eyes from nine patients with type 2 diabetes, aged 55 to 70 years (mean \pm SD: $62.1 \pm 5.16$ years), with mild to moderate nonproliferative diabetic retinopathy graded according to the ETDRS (Early Treatment Diabetic Retinopathy Study) DR grading scale on levels 20 to 35 (presence of microaneurysms, hemorrhages, and/or hard exudates), with little or no clinical evidence of macular edema. Group 3 is composed of 26 eyes of 14 patients, aged 56 to 84 years (mean \pm SD: $75.4 \pm 7.17$ years) with diagnoses of late-stage AMD in one eye and early stage AMD lesions (macular drusen and/or pigmentary changes) in the fellow eye. Eyes with evidence of ocular alterations such as ocular hypertension, glaucoma, media opacities, or retinal disease other than DR or AMD were not included in the analysis.

Best corrected visual acuity was 20/20 for group 1; equal to or better than $20 / 25$ for group 2; equal to or better than 20/40 for early-stage AMD eyes (group 3); and ranging from 20/80 to 20/500 for late-stage AMD eyes (group 3).

Two grading types were used to assess the quality of the final map: a quantitative one and a qualitative one.

The quantitative grading was defined by the mean and SD of the difference in retinal thickness at scan intersections to which two types of errors may contribute. The first is due to registration errors, in which two different retinal locations are brought into alignment by the registration procedure. The second source of errors is due to the erroneous determination of the retinal interfaces from which the retinal thickness is computed. These two sources of errors may be present simultaneously, even at the same scan intersection.

On the other hand, the qualitative assessment of the final mapping takes into account localized abrupt changes of retinal thickness in the map. Although this can be measured by the bending energy of the TPS $^{27}$ (see the last paragraph of the OCT to RT-atlas Registration section in Methods), relatively high values would be found for AMD eye maps compared with those for healthy or DR eyes, resulting from the pathologic condition and not from registration and/or thickness measurement errors.

In this way, retinal thickness maps were classified into one of four grading levels: "poor," "insufficient," "sufficient," and "good." Maps scored poor were those in which notorious abrupt changes were due to registration and/or erroneous thickness determination, leading to local high rough-surface maps. Maps scored as insufficient were those in which retinal thickness map differences at scan intersections led to less rough-surface maps compared with the poor ones. Maps scored as good were those in which the retinal thickness differences at scan intersections were within the normal range (healthy volunteer range), whereas the maps graded sufficient were between the ones graded as insufficient and those graded as good.

\section{Results}

In this study, we were able to establish a mathematical model for the retinal thickness of the healthy human eye by correcting the location and orientation of each individual RTA map. We also made use of $\mathrm{PCA}^{23,26}$ to capture the major shape characteristics of the retinal thickness and therefore the ones that best represent the average population.

Figure 1 shows the computed RT-atlas surface in which the $(0,0)$ in the $x-y$ plane represents the origin of the macular coordinate system (located at the center of the fovea) and the vertical axis is the resulting thickness from the first component of the PCA (i.e., the one corresponding to the higher eigenvalue and therefore having the largest spread of data. It should be noted that this surface represents the average shape of a healthy retina's thickness and not the average thickness). It also clearly shows the asymmetric depression on the temporal side relative to the nasal side, which results from the careful registration of the RTA maps.

This RT-atlas, a statistical shape model (template) defined by 6720 data points, allows us to register OCT scans without resorting to any ocular fundus reference, as specified in OCT to RT-Atlas Registration in the Methods section. A TPS $^{27}$ surface passing through each of these data points is defined by a total of 13,443 parameters.

Likewise, each increased resolution OCT thickness map is now defined by a TPS surface with 2819 parameters.

For each increased-resolution OCT thickness map, the average OCT thickness difference at scan intersections was computed. The mean \pm SD of these averages for the group of healthy volunteers ( $n=12$ eyes) before and after registration through the RT-atlas is $5.93 \pm 1.60$ and $4.17 \pm 1.06 \mu \mathrm{m}$, respectively, which demonstrates the added value of the registration procedure. There was a $30 \%$ reduction of the mean and a $34 \%$ reduction of the SD.

The maps calculated from the healthy eyes and from the DR and AMD eyes were both quantitatively and qualitatively assessed (Table 1), with the healthy eyes group scoring better both quantitatively and qualitatively and the AMD group scoring worse in both classifications.

A similar procedure was followed for each of the patients' eyes $(n=44)$. The average OCT thickness difference at scan intersection was computed, and the mean \pm SD of these average values was reduced $28 \% \pm 24 \%$ due to the registration procedure; these reductions were $35 \% \pm 43 \%$ for the DR patient group and $26 \% \pm 25 \%$ for the AMD patient group.

All thickness maps of healthy eyes were at least classified as sufficient. On the other hand, our technique performed poorly for AMD eyes, as only 2 (7.7\%) of 26 maps were graded sufficient, and none achieved a good grade. Nevertheless, the majority (55.5\%) of DR maps scored good, with only a $0.5-\mu \mathrm{m}$ difference on average from the healthy eyes, and only $11.1 \%$

TABle 1. Average Retinal Thickness Differences at Scan Intersections of Increased-Resolution OCT Thickness Maps, by Classification and Group

\begin{tabular}{lccccc}
\hline \multicolumn{1}{c}{ Group } & Poor & Insufficient & Sufficient & Good & Group Average \\
\hline Healthy volunteers & & & $4.61 / 1.22(n=4)$ & $3.95 / 0.89(n=8)$ & $4.17 / 1.06(n=12)$ \\
Diabetic retinopathy & & $8.78 / 2.55(n=2)$ & $6.12 / 1.54(n=6)$ & $4.50 / 0.94(n=10)$ & $5.51 / 1.98(n=18)$ \\
$\begin{array}{l}\text { Age-related macular } \\
\quad \text { degeneration (AMD) }\end{array}$ & $23.67 / 18.46(n=11)$ & $9.64 / 2.59(n=13)$ & $8.89 / 3.70(n=2)$ & & $15.52 / 14.05(n=26)$ \\
Grade average & $23.67 / 18.46(n=11)$ & $9.52 / 2.60(n=15)$ & $6.08 / 2.45(n=12)$ & $4.25 / 0.96(n=18)$ & \\
\hline
\end{tabular}
to poor.

Data are mean/SD. Note the general increase from the healthy to the AMD patients' group and a similar increase from the groups graded good 


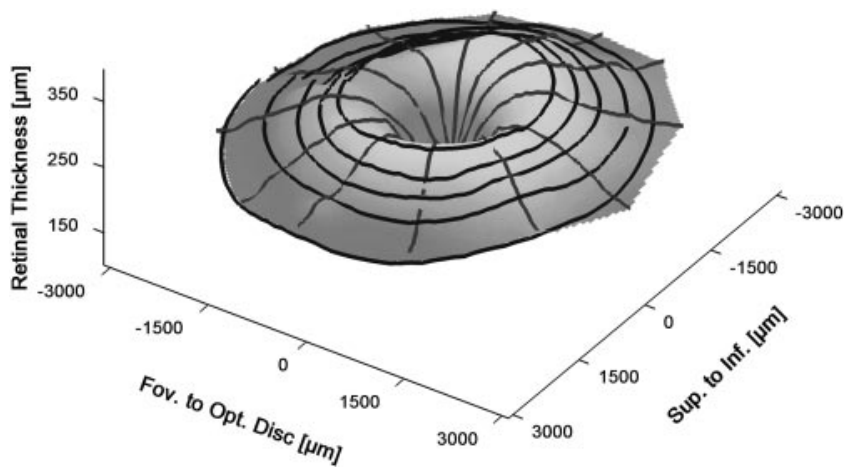

FIGURE 2. 3D view of the increased-resolution OCT thickness map of the right eye of a 30-year-old healthy volunteer shows good superposition of circular and radial line scans, a smooth surface, and good concentricity of the circular scans. This map was scored as good.

scored insufficient, indicating the usefulness of the system for the detailed mapping of retinal thickness in DR eyes.

Figures 2 through 5 show representative cases of the application of the methodology described herein.

The three-dimensional (3D) plot in Figure 2 shows the increased-resolution OCT thickness map for the right eye of a 30-year-old healthy volunteer. The interesting aspects are the smooth surface and the good fixation shown by the concentricity of circular scan lines. The mean \pm SD of the thickness difference at scan intersections is $3.44 \pm 2.99 \mu \mathrm{m}$, and the map was scored good.

Figure 3 shows a 3D plot of a similar map from the left eye of a 69-year-old patient with nonproliferative DR. The noted features are the better definition of the localized retinal edema and the intersection of the circular scan lines. Although it might be considered a registration error, it should be noted that the resulting surface is smooth, and the good agreement on retinal thickness at scan intersections is better than would result if the scans were considered perfectly concentric. This map was scored as good and presents a mean \pm SD thickness difference at scan intersections of $4.76 \pm 4.12 \mu \mathrm{m}$.

Figures 4 and 5 are both from AMD eyes. The 3D plot in Figure 4, the right eye of a 56-year-old patient, shows the irregular surface of the retinal thickness map, plus the much reduced agreement in the retinal thickness at scan intersections (mean $\pm \mathrm{SD}$ of $12.59 \pm 18.42 \mu \mathrm{m}$ ). This map was scored sufficient.

A map of a 78-year-old patient's left eye, graded as poor, is shown in Figure 5, with a mean \pm SD of difference in retinal

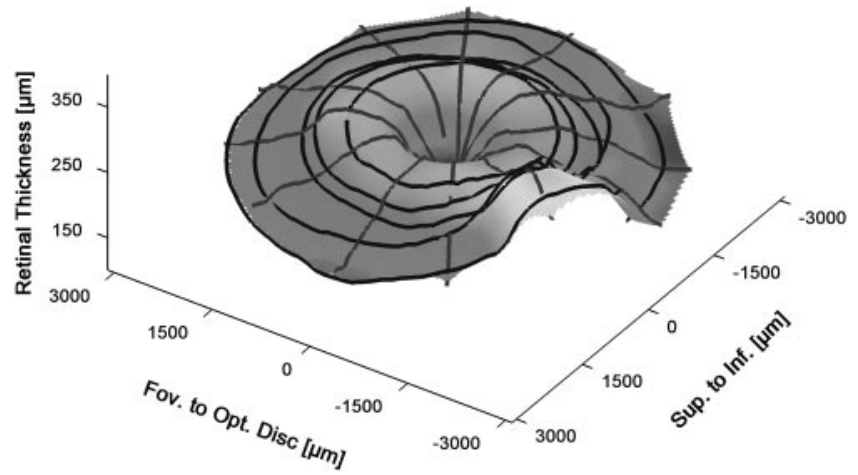

FigURE 3. 3D view of the increased-resolution OCT thickness map of the left eye of a 69-year-old patient with nonproliferative DR shows clear definition of retinal edema at the temporal inferior perimacular area, the good crossing of circular scans, and the agreement of retinal thickness values with the radial scan. This map was scored as good.

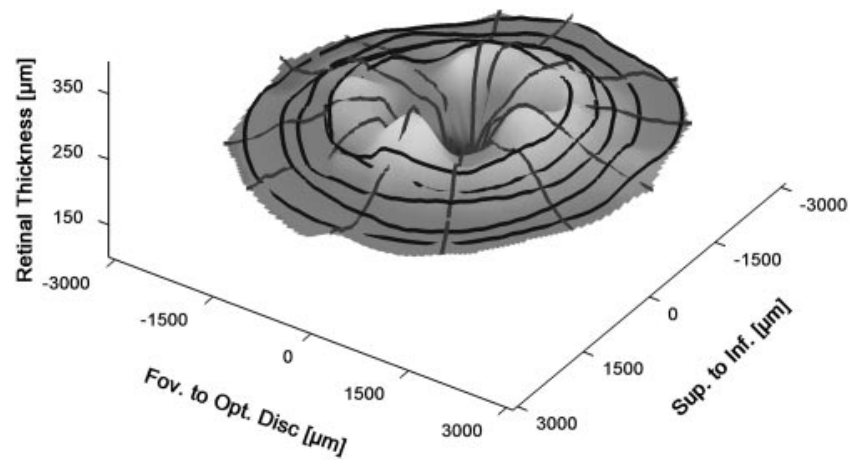

FigURE 4. 3D view of the increased-resolution OCT thickness map of the right eye of a 56-year-old patient with AMD. This map was scored as sufficient.

thickness at scan intersections of $18.73 \pm 24.50 \mu \mathrm{m}$. Two observed prominences resulted from large differences in retinal thickness measurements in relatively small areas.

An additional step was included to allow the integration of non-fast circular scans, according to the standard operating procedure (SOP) defined for the OCT in the EVI-GENORET (European Vision Institute-Functional Genomics of the Retina in Health and Disease) project. Hence, data collected were six-radial-line scans (Fast Macular Protocol) and three-circular scans individually performed. The integration of these two information sources followed an approach similar to that used before, except now the global registration for the circular scans was not considered, which means that steps corresponding to $\hat{\psi}_{1}$ (Eq. 12) and $\hat{\psi}_{2}$ were not performed, at the expense of having larger $\gamma_{3}$ and $\gamma_{4}$ degrees of freedom for the estimation of $\Xi_{1}^{1}, \Xi_{2}^{1}$, and $\Xi_{3}^{1}$. The application of the developed methodology to a 30-year-old healthy volunteer's right eye can be seen in Figure 6, which shows a map that achieved a score of good and a mean \pm SD of $5.16 \pm 4.23 \mu \mathrm{m}$. Although a high average was presented for the differences in retinal thickness at scan intersections, it should be noted that this map was calculated based on a different acquisition protocol (non-fast circular scans). Nevertheless, this value is within $1 \mathrm{SD}$ of the average distribution in the healthy volunteers' group.

\section{Discussion}

Although new OCT devices emerging in the market today do not produce low-resolution maps, because of the high number of A-scans performed, the current systems will be in use for the

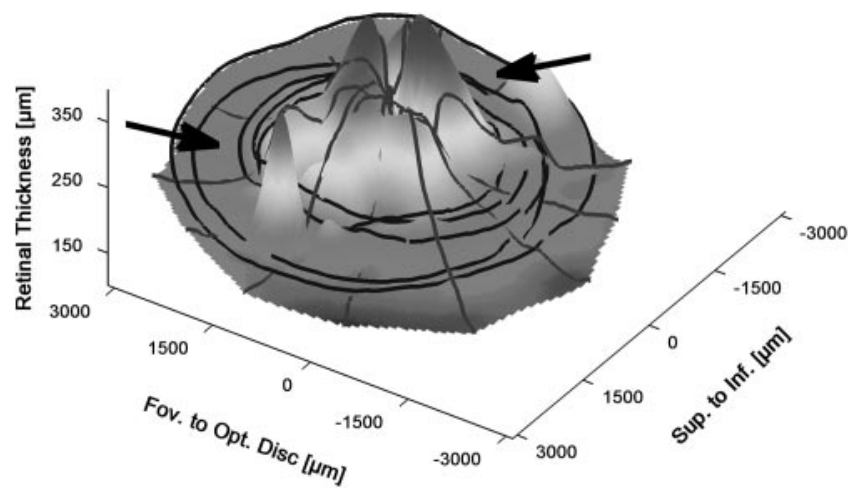

FIGURE 5. 3D view of the increased-resolution OCT thickness map of the left eye of a 78-year-old patient with AMD. Arrows: two prominences resulting from large differences in thickness measurements in relatively small areas. This map was scored as poor. 


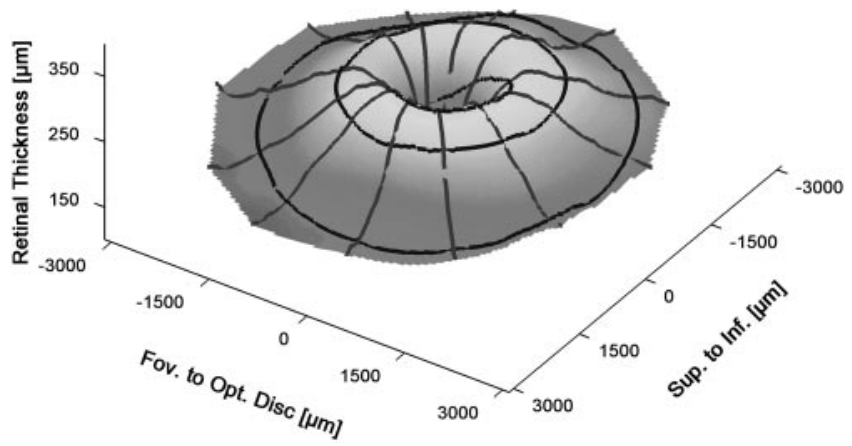

FigURE 6. 3D view of the increased-resolution OCT thickness map of the right eye of a 30-year-old healthy volunteer imaged according to the EVI-GENORET Standard Operating Procedure (SOP).

next decade. It is, therefore, worth using the currently installed capacity to improve the quality of collected data and, in this way, to promote better knowledge of changes in retinal edema due to different diseases.

The system's performance depends on the level of change from the reference (healthy) condition, being tailored to detect initial changes. This finding is clearly demonstrated by the classification of registration scans observed in our healthy volunteers and patients with nonproliferative DR, as opposed to the patients with AMD, for whom the determination of the retinal interfaces frequently failed, and did not provide the registration process with accurate information.

The results indicate that this mapping technique is useful for screening of diseases and the assessment of macular edema in the initial stages of the disease. The monitoring of disease progression/regression may be helpful for clinical trials. However, the mapping technique failed significantly in patients with advanced disease and should not be used in those cases.

A feature previewed for the developed registration process but still not used to its full capacity in this work is the definition of the weighting surface $(\Omega)$, as described in equation 8 . In the present study, this weighting surface was always considered a Gaussian distribution centered on the fovea. Used to its full extent, this surface can be further defined in an interactive process to improve registration for cases clearly far from healthy (e.g., the eyes with AMD considered here). In these cases, a first iteration would be the one described herein.

Finally, it is worth mentioning the increase from 768 to 1408 data points, respectively representing the original OCT map and the increased-resolution map. These additional data points are placed orthogonally to the original ones, which puts most at positions between those on the original radial scans.

\section{References}

1. Lobo C, Bernardes R, Faria de Abreu JR, Cunha-Vaz J. Novel imaging techniques for diabetic macular edema. Doc Ophthalmol. 1999;97:341-347.

2. Lobo C, Bernardes R, Cunha-Vaz J. Alterations of the blood-retinal barrier and retinal thickness in preclinical retinopathy in subjects with type 2 diabetes. Arch Opbthalmol. 2000;118(10):1364-1369.

3. Ciulla T, Armador A, Zinman B. Diabetic retinopathy and diabetic macular edema: pathophysiology, screening, and novel therapies. Diabetes Care. 2003;26:2653-2664.

4. Lobo C, Bernardes R, Faria de Abreu JR, Cunha-Vaz J. One-year follow-up of blood-retinal barrier and retinal thickness alterations in patients with type 2 diabetes mellitus and mild nonproliferative retinopathy. Arch Ophthalmol. 2001;119(10):1469-1474.
5. Cunha-Vaz J, Bernardes R, Lobo C, Faria de Abreu JR. Measurements of retinal thickness for evaluation of macular edema. Contemp Opbthalmol. 2002;1(25):1-6.

6. Lobo C, Bernardes R, Figueira J, Faria de Abreu JR, Cunha-Vaz J. Three-year follow-up study of blood-retinal barrier and retinal thickness alterations in patients with type 2 diabetes mellitus and mild nonproliferative diabetic retinopathy. Arch Opbthalmol. 2004;122:211-217.

7. Lobo C, Faria P, Soares M, Bernardes R, Cunha-Vaz J. Macular alterations after small-incision cataract surgery. $J$ Cataract Refract Surg. 2004;30(4):752-760.

8. Strøm C, Sander B, Larsen N, Larsen M, Lund-Andersen H. Diabetic macular edema assessed with optical coherence tomography and stereo fundus photography. Invest Ophthalmol Vis Sci. 2002; 43(1):241-245.

9. Shahidi M, Ogura Y, Blair N, Rusin M, Zeimer R. Retinal thickness analysis for quantitative assessment of diabetic macular edema. Arch Opbthalmol. 1991;109:1115-1119.

10. Hee M, Puliafito C, Duker J, et al. Topography of diabetic macular edema with optical coherence tomography. Ophthalmology. 1998; 105:360-370.

11. Zeimer R, Shahidi M, Mori M, Zou S, Asrani S. A new method for rapid mapping of the retinal thickness at the posterior pole. Invest Opbthalmol Vis Sci. 1996;37(10):1994-2001.

12. Gieser J, Rusin M, Mori M, Blair N, Shahidi M. Clinical assessment of the macula by retinal topography and thickness mapping. Am J Ophthalmol. 1997;124(5):648-660.

13. Landau D, Schneidman E, Jacobovitz T, Rozenman Y. Quantitative in vivo retinal thickness measurements in healthy subjects. $O p b$ thalmology. 1997;104(4):639-642.

14. Bouma B, Tearney G. Handbook of Optical Coberence Tomography. New York: Marcel Dekker; 2002.

15. Schuman J, Puliafito C, Fujimoto J. Optical Coherence Tomography of Ocular Diseases. Thorofare, NJ: Slack; 2004.

16. Ciulla T, Regillo C, Harris A. Retina and Optic Nerve Imaging. Philadelphia: Lippincott Williams \& Wilkins; 2003.

17. Schmitt J. Optical coherence tomography (OCT): a review. IEEE J Sel Top Quantum Electron. 1999;5(4):1205-1215.

18. Pedut-Kloizman T, Pakter H, Schuman J, Szwartz J, Hee M. Ophthalmic diagnosis using optical coherence tomography. Opbthalmol Clin North Am. 1998;11(3):465- 486.

19. Hee M, Izatt J, Swanson E, Huang D, Schuman J, Lin C, Puliafito C, Fujimoto J. Optical coherence tomography of the human retina. Arch Opbthalmol. 1995;113:325-332.

20. Polito A, Shah S, Haller J, et al. Comparison between retinal thickness analyzer and optical coherence tomography for assessment of foveal thickness in eyes with macular disease. Am J Ophthalmol. 2002;134(2):240-251.

21. Pires I, Bernardes R, Lobo C, Soares M, Cunha-Vaz J. Retinal thickness in eyes with mild nonproliferative retinopathy in patients with type 2 diabetes mellitus: comparison of measurements obtained by retinal thickness analysis and optical coherence tomography. Arch Ophthalmol. 2002;120:1301-1306.

22. Diedrichsen J. A spatially unbiased atlas template of the human cerebellum. Neuroimage. 2006;33:127-138.

23. Heitz G, Rohlfing T, Maurer Jr C. Statistical shape model generation using nonrigid deformation of a template mesh. In: Medical Imaging 2005: Image Processing. Vol 5747. Bellingham, WA: SPIE; 2005;1411-1421

24. Jaume S, Ferrant M, Macq B, et al. Tumor detection in the bladder wall with a measurement of abnormal thickness in CT scans. IEEE Trans Biomed Eng. 2003;50(3):383-390.

25. Joshi S, Davis B, Jomier M, Gerig G. Unbiased diffeomorphic atlas construction for computational anatomy. Neuroimage. 2004;23: S151-S160.

26. Petrou M, Bosdogianni P. Image Processing: The Fundamentals. Hoboken, NJ: John Wiley \& Sons, 1999.

27. Costa L, Cesar R Jr. Shape Analysis and Classification: Theory and Practice. Boca Raton, FL, CRC Press; 2000. 